\title{
Optical coherence tomography angiography in diabetic retinopathy: an updated review
}

\author{
Zihan Sun ${ }^{1} \cdot$ Dawei Yang ${ }^{1} \cdot$ Ziqi Tang ${ }^{1} \cdot$ Danny S. Ng $^{1,2} \cdot$ Carol Y. Cheung ${ }^{1}$
}

Received: 26 August 2020 / Revised: 27 September 2020 / Accepted: 15 October 2020 / Published online: 24 October 2020

(c) The Author(s), under exclusive licence to The Royal College of Ophthalmologists 2020

\begin{abstract}
Diabetic retinopathy (DR) is a common microvascular complication of diabetes mellitus. Optical coherence tomography angiography (OCTA) has been developed to visualize the retinal microvasculature and choriocapillaris based on the motion contrast of circulating blood cells. Depth-resolved ability and non-invasive nature of OCTA allow for repeated examinations and visualization of microvasculature at the retinal capillary plexuses and choriocapillaris. OCTA enables quantification of microvascular alterations in the retinal capillary network, in addition to the detection of classical features associated with DR, including microaneurysms, intraretinal microvascular abnormalities, and neovascularization. OCTA has a promising role as an objective tool for quantifying extent of microvascular damage and identify eyes with diabetic macular ischaemia contributed to visual loss. Furthermore, OCTA can identify preclinical microvascular abnormalities preceding the onset of clinically detectable DR. In this review, we focused on the applications of OCTA derived quantitative metrics that are relevant to early detection, staging and progression of DR. Advancement of OCTA technology in clinical research will ultimately lead to enhancement of individualised management of DR and prevention of visual impairment in patients with diabetes.
\end{abstract}

\section{Introduction}

Diabetes mellitus (DM) is one of the world's fastest-growing chronic diseases. It is estimated that the number of people with DM will increase to 642 million by 2040 [1]. Diabetic retinopathy (DR) is a common and specific microvascular complication of DM that lead to progressive visual impairment and even blindness. The prevalence of DR ranges reportedly from $30 \%$ to $45 \%$ among DM, and 1 in 10 has vision-threatening DR [2]. With a projected increase in the incidence of DM, increased life expectancy and an ageing population, it is anticipated that the impact of vision impairment associated with DR will inevitably increase in the coming years. The diagnosis of DR is based on clinical

$\triangle$ Carol Y. Cheung

carolcheung@ cuhk.edu.hk

1 Department of Ophthalmology and Visual Sciences, The Chinese University of Hong Kong, Hong Kong Special Administrative Region, China

2 Hong Kong Eye Hospital, Hong Kong Special Administrative Region, China fundoscopic examination. It is broadly divided into two categories: non-proliferative diabetic retinopathy (NPDR), and proliferative diabetic retinopathy (PDR) which is associated with the development of neovascularization (NV) [2]. The main sight-threatening complications of DR are diabetic macular oedema (DMO), diabetic macular ischaemia (DMI), and PDR $[2,3]$. There are a growing number of imaging modalities that can be used in the screening, evaluation, diagnosis, and treatment of DR. Currently, the standard to assess the extent of vascular leakage and presence of ischaemia is dye-based fluorescein angiography.

With technological advances, optical coherence tomography angiography (OCTA), may serve as a potential rapid, non-invasive image modality as an adjunct for assessing microvascular changes in capillary level $[4,5]$. It is performed by acquiring repeated OCT B-scans at the same location in order to detect changes in reflectance signal from the movement of red blood cells through blood vessels in the volumetric OCT scans. It allows depthresolved visualization of the retinal microvasculature by selecting different en face slabs from different retinal layers without intravenous dye injection. Microvascular changes in the superficial, intermediate, and deep capillary plexuses can be evaluated individually. 
In this article, we aim to provide an updated overview of the current applications of OCTA in the evaluation of DR. We conducted a literature search via PUBMED database for articles written in the English language until Jun 1, 2020, with the following medical subject headings: "OCTA"; "OCT angiography"; "Diabetic Retinopathy" or "Diabetes."

\section{Drawbacks of fluorescein angiography}

Fluorescein angiography (FA) is an invasive imaging technique that requires intravenous administration of dye and imaging up to 10 to $30 \mathrm{~min}$ [6]. It provides two-dimensional images that allow for dynamic visualization of blood flow with a wide-field of view. Although the fluorescence enabled visualization of retinal capillaries, it cannot separately visualize the intra-retinal structures of the major capillary networks. Specifically, fluorescein angiograms mainly corresponded to the superficial retinal vessels, whereas the deeper retinal capillaries were not visualized in the fluorescein angiograms, possibly because of light scattering in the retina [7-9]. Similarly, FA has very limited ability to visualize the choroidal vasculature, which may also be affected in DM eyes. Patterns of dye leakage, pooling, and staining can be appreciated from FA [10]. However, the ability to detect microvasculature alterations has also been limited by the leakage in FA and superposition of the capillary networks. For DM eyes, FA remains the gold standard for detecting vascular leakage, DMI, as well as retinal NV. Since FA is invasive, relatively expensive, and time-consuming, it is not an ideal technique to use regularly in clinical practice. Furthermore, although FA is generally considered as a safe procedure, the dye still poses risks ranging from nausea to allergic reactions, including anaphylaxis in rare occasions. A non-invasive imaging technique would be ideal for patients who require frequent followup exams or those that cannot tolerate the adverse effects of dye injection.

\section{Advantages of OCTA}

OCTA is based on mapping red blood cells movement over time from volumetric OCT scans. At each B-scan position, scan is repeated to detect the motion contrast. The degree of motion contrast corresponds to angiographic flow, as the only expected motion in the retina is blood flow in vessels [5]. Although OCTA technology cannot show leakage from the blood vessels (unlike FA), it has several advantages compared with traditional FA. First, it is non-invasive. OCTA techniques allows visualization and detailed assessment of changes in retinal microvasculature without dye injection. This is very important as OCTA can be obtained more frequently than FA for managing patients' eyes longitudinally. Second, OCTA data acquisition is more rapid than FA. OCTA is developed based on the conventional OCT and it is available on regularly used OCT platform. Furthermore, OCTA data is three dimensional and depth resolved. Individual capillary plexuses can be visualized and assessed. Improved software algorithm automatically generates the images of the superficial and deep capillary plexuses, and the user can further adjust and customize the segmentation of the retinal vasculature to obtain images of other layers such as the intermediate capillary plexuses, which helps to visualize pathological features that are not available in traditional dye-based angiography.

\section{Morphological changes of DR signs in OCTA}

Several morphological features of DR can be detected by OCTA, including microaneurysms (MA), intraretinal microvascular abnormalities (IRMA) and NV, offering additional information to the localization of these microvascular changes.

\section{Microaneurysms (MA)}

MA, identified clinically by ophthalmoscopy as deep-red dots varying from 25 to $100 \mu \mathrm{m}$ in diameter, are usually the first visible sign of DR. Previous studies have already showed that increased number of MA and MA turnover are associated with a higher risk for DR progression and DMO [11-13]. By using OCTA, Thompson et al. demonstrated that OCTA could pick up MAs even not shown on a dilated clinical examination [14]. Moreover, the depth-resolved ability of OCTA allows precise localization of MAs. Ishibazawa and associates showed that the MAs were located mainly in the deep plexus identified by OCTA [15]. Moreover, Schwartz et al. and Ishibazawa et al. demonstrated that OCTA could detect MAs that are not detectable on FA $[15,16]$.

However, a number of studies showed that not all MAs detected by FA could be visualized with OCTA, which may be influenced by blood flow turbulence within the MAs [15, 17-21]. Histopathologic studies have reported that the lumen configuration in MAs consists of diverse components such as thickened, hyalinized, fibrous, laminated, and lipidcontaining basement membrane, as well as hypercellular or multilayered endothelial cells [22]. Studies have shown a correlation between the MA's reflectivity and its detectability on OCTA - MAs with higher reflectivity are more likely to be detected [23]. Nevertheless, it is still unclear whether OCTA is comparable to FA in terms of detecting MAs.

\section{Intraretinal microvascular abnormalities (IRMA)}

IRMA is one of the defining features of severe NPDR based on the "4-2-1" criteria from the Early Treatment Diabetic 


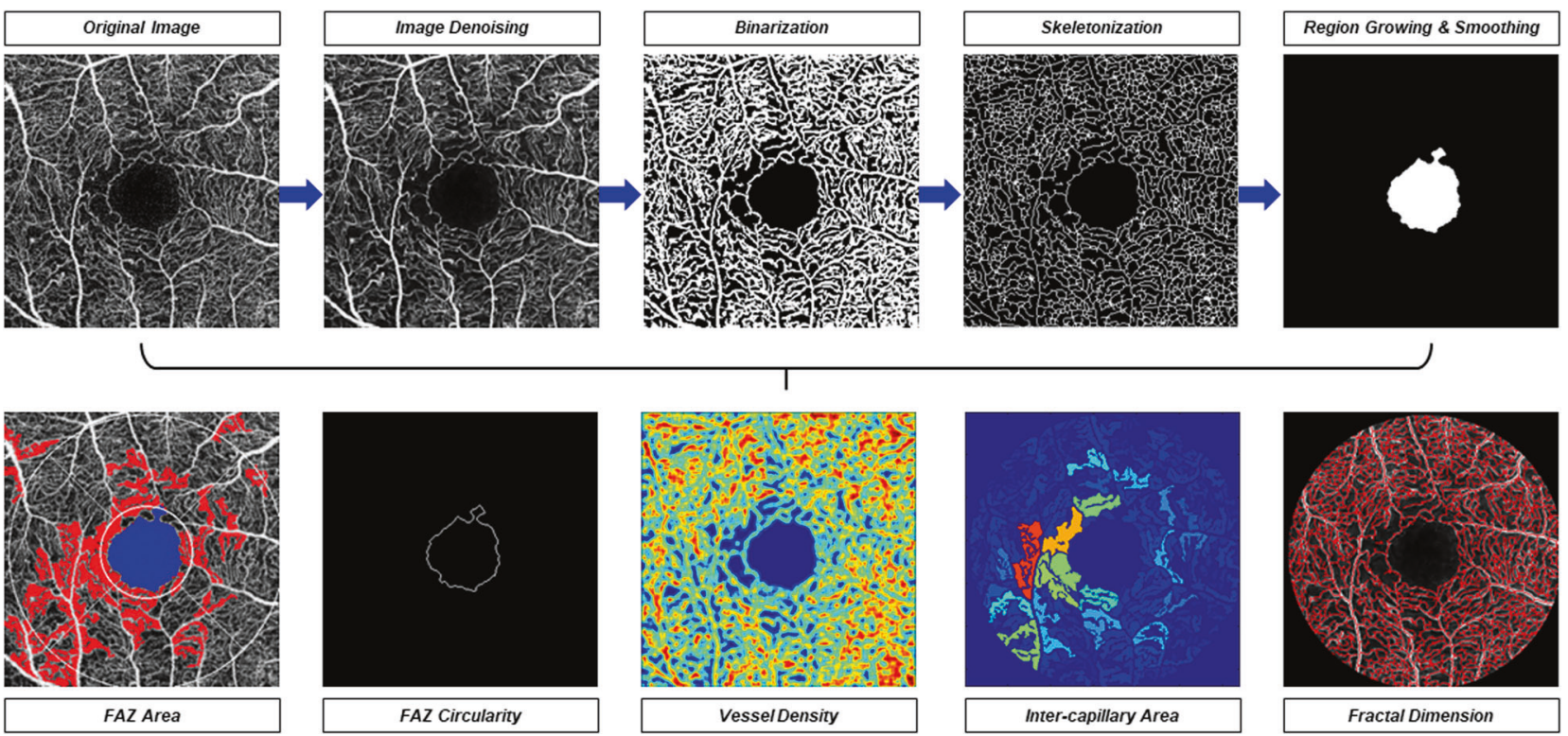

Fig. 1 Quantification of retinal microvasculature from OCT angiography (OCTA) images. After applying different imaging processing steps including denoising, binarization, skeletonization, region growing and smoothing, a series of OCTA metrics including

Retinopathy Study (ETDRS) [24]. IRMAs are shunt vessels due to abnormal branching or dilation of existing capillaries within the retina. The formation of IRMA helps to supply areas of non-perfusion in DR. Nevertheless, the conventional method for detection of IRMA on colour fundus photography poses great challenge to clinicians. OCTA with conventional OCT B-scan has the advantage of displaying both en face and cross-sectional views to assist differentiation of IRMAs from NV. On OCTA, IRMAs appear as abnormal, branching, dilated retinal vessels that do not protrude into the vitreous [25]. Schaal et al. have reported a higher detection rate of IRMAs on OCTA than colour fundus photography [26]. In addition, OCTA may enable the classification of IRMA into more detailed. Akito et al. observed morphological changes in IRMAs before and after photocoagulation and proposed a classification system via OCTA [27]. The changes in the IRMAs were divided into five subtypes: unchanged; tuft regression; reperfusion; mixed (combined tuft regression/reperfusion); and worsening (new appearance of tuft). They suggested that some types of IRMAs had aspects of remodelling; some types had aspects of NV. Taken together, OCTA might present a more severe state of DR than suspected by colour fundus grading alone, and the introduction of a new grading system of DR based on OCTA findings may be considered in the future.

\section{Neovascularization (NV)}

Proliferative diabetic retinopathy (PDR) is the advanced stage of DR and is characterized by the development of abnormal foveal avascular zone (FAZ) area, FAZ circularity, vessel density, inter-capillary area and fractal dimension were automatically calculated by a customized MATLAB (MathWorks, Natick, MA) programme.

blood vessels - neovascularization - which may be of the disc (NVD) or elsewhere (NVE), secondary to retinal ischaemia [28]. If left untreated, NV results in haemorrhagic and tractional complications, which may substantially deteriorate patients' vision [29]. Therefore, early detection of NV is essential to facilitate early intervention. The ETDRS established the current standard method of assessing NV seen on stereoscopic colour fundus photographs [30]. Retinal NVs are detectable on OCTA via observation of flow signal above the ILM $[25,28]$. Recent studies showed that OCTA can detect early retinal NVs and identify the origins and morphological patterns of NVs in PDR, hence allowing classification of the lesion, offering a better understanding of the pathophysiology and helps to guide the management strategies [21, 25]. OCTA may also detect subtle NVs. Hagar et al. evaluated the utility of wide-field OCTA compared with clinical examination in diagnosing PDR [31] and found that wide-field OCTA has a higher detection rate of PDR than clinical examination, suggesting that wide-field OCTA could be used for early detection and characterization of retinal NV.

\section{Quantification of microvascular alterations from OCTA images}

Quantification of OCTA images in DR was firstly described by Jia and associates in 2015 [32]. After that, a number of quantitative metrics have been developed and correlated with DM and DR over last few years. Figure 1 shows an example of image processing methods (e.g., image 
denoising, binarization, skeletonization) for quantification of retinal microvasculature from OCTA image using a customized MATLAB programme [33].

\section{Foveal avascular zone (FAZ) measurements}

The human foveola, a rod-free region of the central retina, is responsible for central vision, as it has the maximum cone photoreceptor packing density. The absence of vasculature and the overlaying inner retinal tissue are believed to maximize the optical quality by reducing light scattering. This central avascular region is known as the foveal avascular zone (FAZ) [34]. In DR, enlargement of FAZ occurs due to the loss of capillaries in the adjacent vessels [35]. Therefore, the most common approach is to measure the area of the FAZ. FAZ area is believed to be a measurement that can indicate diabetic microvascular changes. In addition, other metrics have also been adopted to measure the FAZ, such as the FAZ perimetry, FAZ radius and FAZ circularity. Shiihara et al. have suggested that the shape might be better to characterize the FAZ because heterogeneity exists in the size of FAZ among the normal population [36]. Nonetheless, a wide range of variation of FAZ area (from $0.071 \mathrm{~mm}^{2}$ to $0.527 \mathrm{~mm}^{2}$ ) had been reported in healthy eyes, whose visual acuities were recorded as 20/20 [37] and enlarged FAZ area was correlated with shorter axial length [33]. Assessment of FAZ circularity might be more informative of disease-induced microvascular changes than the FAZ area, as the shape of FAZ was generally spherical or somehow ellipses in normal eyes. It is convincing that once the obstruction of the innermost capillaries surrounding the fovea occurs, the FAZ becomes irregular in shape. Hence, FAZ circularity on OCTA also serves as an indicator of capillary dropout and macular ischaemia.

\section{Vessel density}

Vessel density (VD) is generally defined as the proportion of blood vessel area over the total measured area based on the binarized image for indicating the perfusion of the retinal microvasculature [38]. It is noteworthy that VD varies with age and gender [39], and corrected with retinal layer thickness, such as macular ganglion-cells inner plexiform layer [33, 40]. In addition, VD is highly correlated with signal strength (SS) [41]. Careful interpretation of OCTA-measured VD is recommended, especially images with poor quality.

\section{Vascular length density (VLD)/skeleton density (SD)}

Vessel length density (VLD) or skeleton density (SD) is proposed to serve as a counterpart of VD which quantifies the vessel density by only considering whether the vessel exists per unit area, regardless of the vessel diameters [42]. Since every vessel represents a single-pixel line, large vessels and small capillaries contribute equally to the VLD quantification. Consequently, compared with VD, VLD is thought to more sensitive to the perfusion changes at the capillary level [43].

\section{Vessel diameter index (VDI)}

The vessel diameter index (VDI) is calculated as the area occupied by blood vessel from the binarized image over the total length of blood vessel from the skeletonized image, representing the average vessel calibre of blood vessels [44]. Increased VDI is shown to be correlated with higher fasting glucose level [33].

\section{Fractal dimension}

Fractal dimension (FD) measures the complexity of a vasculature branching pattern. FD is calculated from a skeletonized line tracing using the box-counting method, which divides each image into a series of squares for various side lengths and the number of boxes is counted.

\section{Vessel tortuosity}

Retinal vessel tortuosity is defined as the integral of the curvature square along the path of the vessel, normalized by the total path length. Vessel tortuosity was previously measured from fundus photographs using computer-assisted programmes $[45,46]$. Patients with DM were found to have increased vessel tortuosity as compared to healthy controls, suggesting that vessel tortuosity may be an early indicator of vascular damage to the retina [47].

\section{Quantitative OCTA metrics in diabetic retinopathy and diabetic macular oedema}

\section{OCTA may reveal DR before it is clinically detectable}

Several studies have shown the capability of OCTA in detecting subclinical DR (Table 1). Cao and associates reported a decreased parafoveal VD in eyes without clinically detectable DR compared to healthy controls [48]. Yashi et al. [49] observed an increase in the FAZ area in diabetic subjects compared with controls, which is in concordance with previous literature [50-52]. In addition to the above-mentioned cross-sectional studies, Sun et al. also showed that certain OCTA metrics could predict the incidence of DR in DM patients in a cohort study [53]. They included 66 eyes without DR at baseline and found 


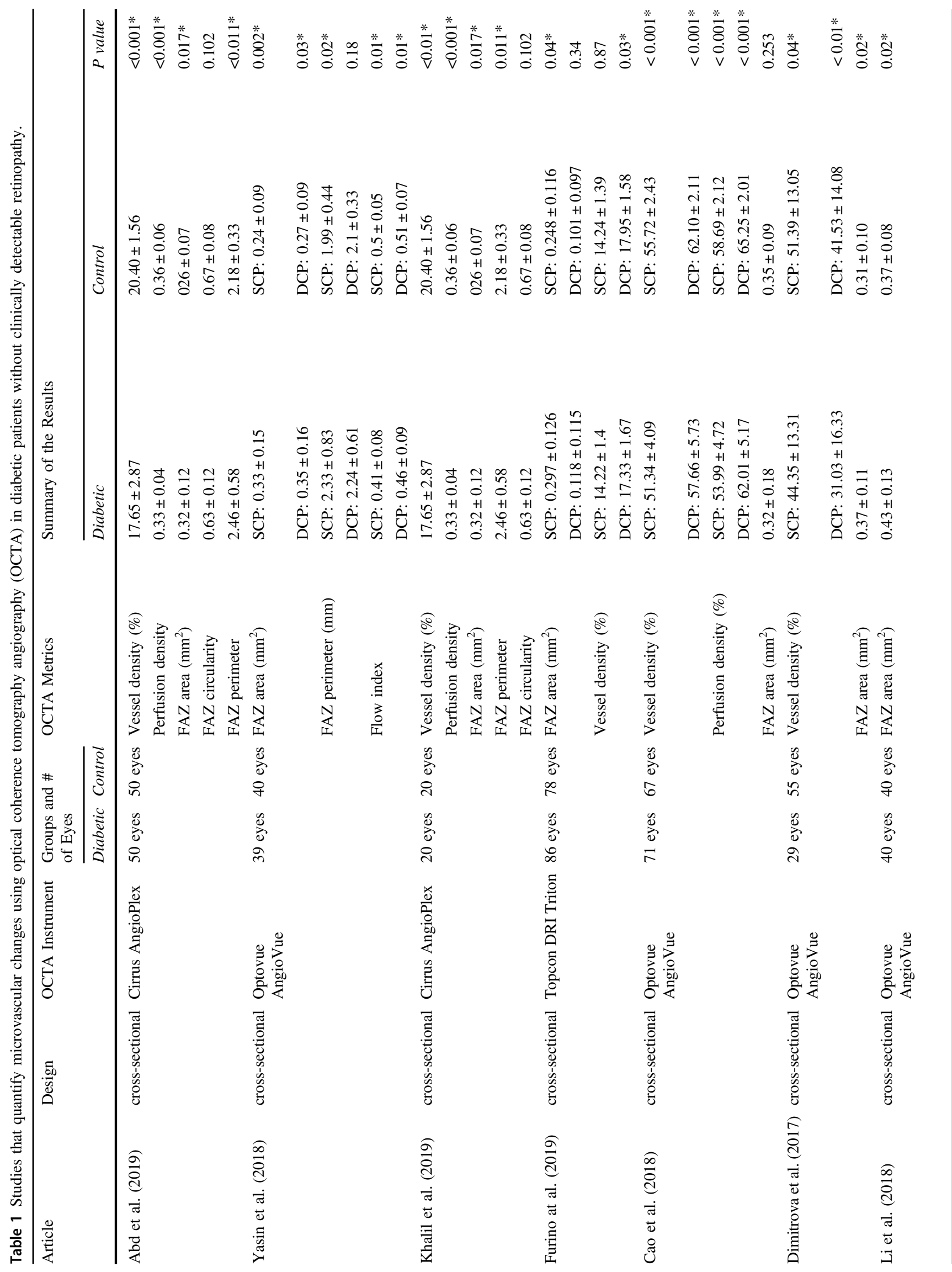




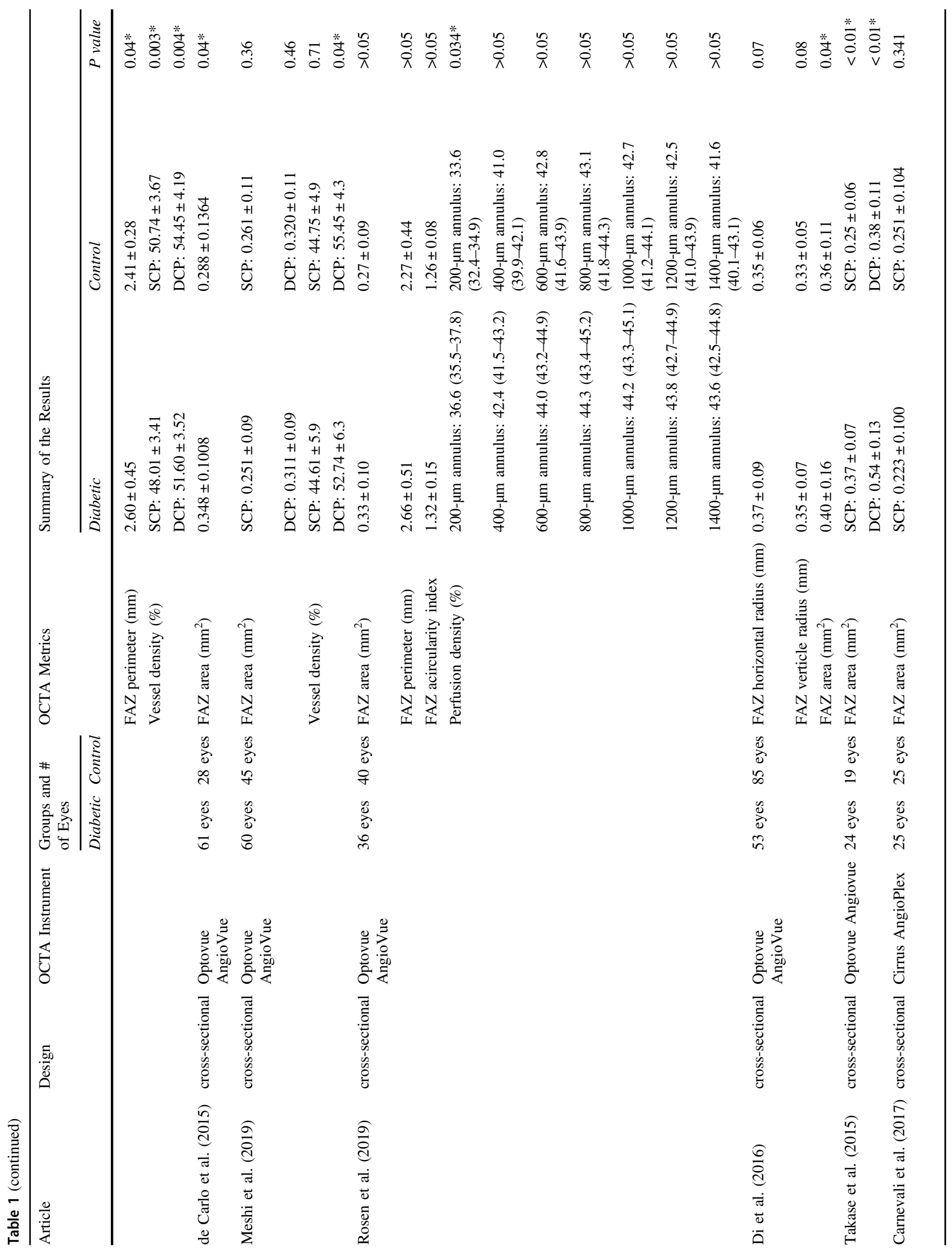




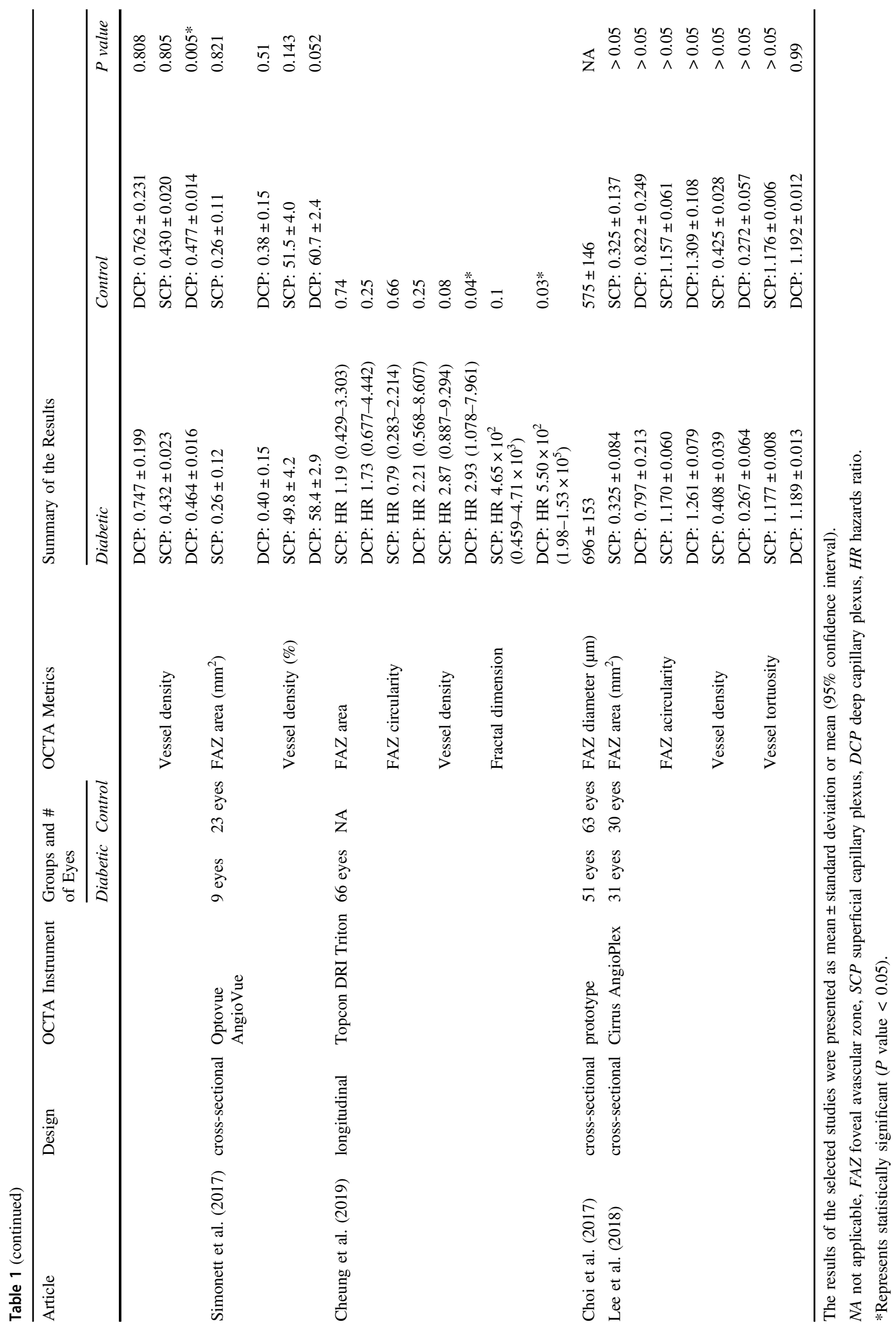


that eyes with lower VD and lower FD of the DCP were at higher risk of developing DR. This longitudinal study adds to several recently published cross-sectional studies and suggests that OCTA metrics may be useful in early detection and prediction of DR.

\section{Quantitative metrics are correlated with severity of DR}

Numerous studies demonstrated quantitative OCTA metrics on SCP and DCP are correlated with severity of DR, suggesting that the extent of microvascular damage (e.g., DMI) in individual capillary plexus can be quantified from OCTA [33, 54-61]. In summary, these studies generally reported that enlarged FAZ area, decreased FAZ circularity, lower VD, increased VDI, decreased FD, and increase vessel tortuosity are significantly associated with worsening DR. For example, Kim et al showed that decreased VD, SD, FD, and increased VDI are associated with worsening DR. Tang et al found that enlarged FAZ area, decreased FAZ circularity, lower VD and decreased FD on SCP, enlarged FAZ area and lower VD on DCP, were significantly associated with worsening DR. A recent systemic review conducted by Johannesen et al. [62] demonstrated that FAZ area is larger in NPDR and in PDR compared with the control group.

\section{Quantitative OCT metrics are associated with DR progression}

Currently, little is known about the predictive value of OCTA metrics on DR progression because the study design in most of the current literature is cross-sectional. Sun [53] and associates determined the predictive value of OCTA in DR progression. Of note, only metrics (FAZ area, VD, and FD) at the DCP showed significant associations with the risk of DR progression, whereas metrics at the SCP did not show similar results. These findings are in line with previous FA studies that DMI (e.g., clinically defined as enlargement of FAZ and capillary dropout in parafoveal area) are associated with progression of DR and support previous cross-sectional findings, emphasizing the important role of the deep capillary plexus in DR. The DCP may be more susceptible to ischaemic damage because it may reside in a watershed zone, where the deep layer of the retinal circulation sits next to high oxygen requirements of the outer plexiform layer [63]. Previous histologic studies also indicated that the DCP is more vulnerable to injury and preferentially affected [64]. Taken together, quantitative OCTA analysis, indicative of DMI likely, may identify DM individuals at risk of developing DR progression independently.

\section{OCTA in diabetic macular oedema (DMO)}

Despite OCT can illustrate structural changes prominently and aid in detecting cystic spaces in DMO, OCTA has low reliability in visualizing the capillary networks in eyes with DMO [65]. This is because the accumulated fluid may affect the segmentation capabilities of OCT, and in turn, the incorrect segmentation may lead to a false interpretation of OCTA images. In addition, DMO has an inverse association with OCTA signal intensity as the fluid weakens the reflected signal from the deep layer [66, 67]. It is also reported that the rate of flow voiding does not precisely match with the cystic space as the fluid may compress the vessels, thus decreasing flow below the detection limits of the OCTA algorithms [67].

To overcome the segmentation issues, Lee et al. manually adjusted the SCP/DCP boundary in the eyes with severe DMO, and demonstrated that patients with DMO exhibit significant damage in the DCP rather than SCP [65]. They also noted that, compared with DMO eyes that responded well, poor responders showed extensive damage to the integrity of the DCP, but not the SCP. This finding revealed that the DCP might be critical for the treatment response to anti-VEGF therapy, suggesting that the extent of DCP loss assessed by OCTA could be a useful biomarker for predicting the treatment response of DMO.

Apart from this, Sun et al. longitudinally investigated the association between OCTA metrics and DMO development among eyes without DMO at baseline visit [53]. VD of the SCP was reported to be associated with the development of DMO over 2 years of follow-up, suggesting that quantitative OCTA analysis may identify DM individuals at risk of developing DMO. Further studies are needed to determine whether measurement of OCTA metrics can identify a more specific subgroup of patients who could benefit from more intensive investigations or even start treatment.

\section{OCTA of the choroidal vascular changes in diabetic eyes}

The choroidal circulation is the major source of oxygen and nutrients for the choroid and the outer retina [68]. Accordingly, the assessment of choroidal blood flow is essential to understand the pathogenesis of diabetic eye diseases. The choroid is mainly composed of vessels and stroma (extravascular tissue). Most choroidal space is occupied by vessels differentiated in three vascular layers the choriocapillaris (CC), the Sattler's layer, and Haller's layer [68]. Measuring the choroidal blood flow remains challenging by using traditional dye-based angiography. The advent of OCTA makes it possible to visualize and quantify the choroidal vasculature, particularly the CC. 
Several groups have studied the CC perfusion in diabetic eyes and yielded discordant findings. Choi et al. described focal and diffuse CC flow impairments in diabetic eyes [68]. Nesper and associates reported an increased CC nonperfusion area in diabetic eyes without retinopathy compared with the control group [55]. However, other groups reported no significant difference in $\mathrm{CC}$ vessel density between diabetic eyes without retinopathy versus healthy controls $[40,69]$. There has been concerns about the insufficient resolution of commercial OCTA systems to measure CC because the $\mathrm{CC}$ is extremely dense in the posterior pole with small intercapillary distances $(5-20 \mu \mathrm{m})$ that are smaller than the OCT system's lateral resolution $(15-20 \mu \mathrm{m})$ [70]. Therefore, researchers have proposed to use the flow deficit to analyse CC perfusion [71]. The flow deficit represents the area where there is a lack of CC flow or CC flow below the OCT system's detectable threshold [72]. Dai and associates have reported increase $\mathrm{CC}$ flow deficits in diabetic eyes without retinopathy compared to age-matched controls [73]. This finding echoes with previous histopathological studies, in which CC dropouts were more pronounced in postmortem subjects with DM than those without DM [74].

\section{Experimental OCTA studies}

A correct interpretation of OCTA images requires good knowledge of the normal vascular architecture of the retina. Although differences exist in retinal vasculature between humans and rodents, applications of OCTA are not confined to human subjects. There are few OCTA studies on experimental mice have been reported [75-77]. For example, Uehara et al. reported that OCTA successfully detects age-dependent retinal vasculature alterations in type 1 diabetic Ins $2^{\text {Akita/+ }}$ mouse. The superficial and intermediate retinal vasculature trended toward a relatively higher density in as early as 2 months of age. At 6 months of age, Ins $2^{\text {Akita/+ }}$ mice showed a significant decrease of deep retinal vasculature compared to the other groups. This finding supports the potential of OCTA being useful in studying DR using animal models. Furthermore, OCTA makes it possible to conduct longitudinal monitoring of vascular alterations, which in turn leads to the possibility of analysing the effects of pharmacological treatments in the future.

\section{Artefacts and limitations of OCTA}

Image artefacts in OCTA are common [78]. Generally, image artefacts in OCTA occur as a result of one or more of the following aspects: [1] the scanning methodology used to generate the motion contrast signal [2], data processing [3],

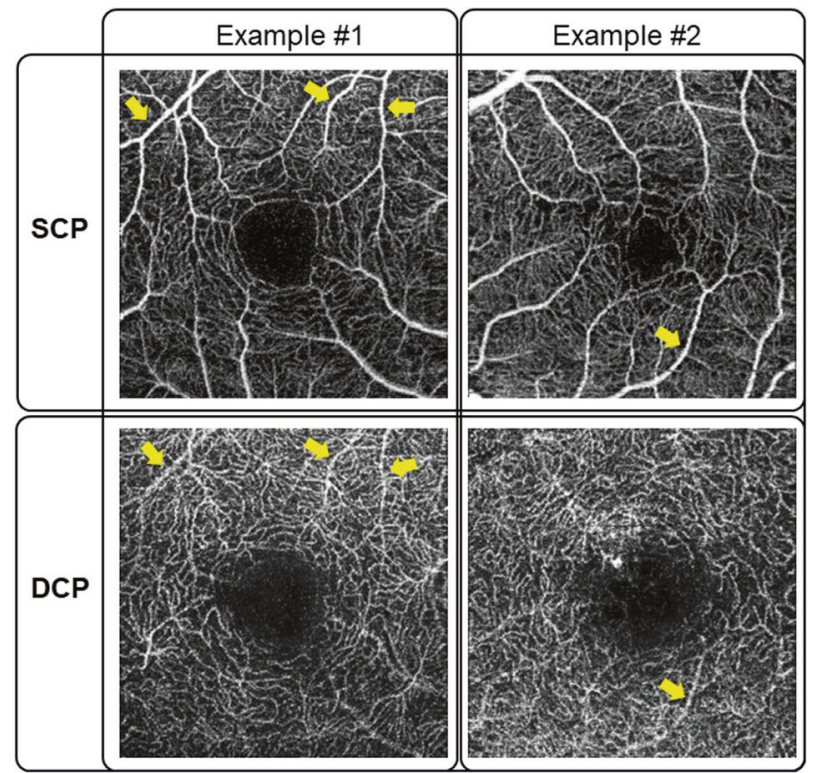

Fig. 2 Examples of shadowgraphic projection artefacts from superficial capillary plexus (SCP) on the deep capillary plexus (DCP) (indicated by yellow arrows). These "false" blood flow signals interfere with the visualization of deeper capillary plexus and lead to incorrect interpretations.

movement of the eye, and [4] the intrinsic properties of the eye and pathology [79]. Given the 3D nature of OCT, the fluctuating shadows from flowing blood cells in the superficial vessels also cast extra flow signals to the deeper vascular networks, generating false vessel networks when producing the en face flow images of deeper retinal tissue. Figure 2 shows examples of shadowgraphic projection artefacts from SCP on DCP.

These "false" blood flow signals interfere the visualization of deeper retinal plexuses (ICP and DCP) and CC, and lead to incorrect interpretations [80]. Projection removal software has been designed to overcome this issue; however, the current technology mainly works by subtracting the superficial OCTA image from the deep ones, which may inevitably lose information from the deep layer (Fig. 2). Other limitations of OCTA include its limited field of view, inability to view leakage, prone to have artefacts, and inability to detect blood flow below the slowest detectable threshold. Another major issue is the lack of consensus on segmentation criteria. This may partially explain the inconsistency among different OCTA devices. Segmentation strategy should be further discussed.

\section{Using deep learning to interpret OCTA images for DR}

Deep learning (DL) is a class of machine learning technique that allows computational models composed of different 
processing layers to learn representations of data with multiple levels of abstraction. Compared with conventional methods, DL has been shown to its superiorities in saving tremendous workforce and financial resources and achieving higher accuracies in many domains [81-84]. With the robust diagnostic performance in detecting various pathological conditions, DL has been applied to ocular imaging, principally fundus photographs and OCT, to screen major ophthalmic disease such as DR, age-related macular degeneration and glaucoma, of which either has wellestablished guidelines and/or requires long-term follow-up [85-89]. OCTA, as a still new auxiliary diagnostic tool, though it has not yet been widely used in disease screening or classification, various convolutional neural networks models have been developed for OCTA related-tasks.

In 2018, Guo et al. developed a DL CNN, with a powerful multi-scale feature extraction capability incorporated to segment the non-perfusion area (NPA) from OCTA image with $6 \times 6 \mathrm{~mm}^{2}$. Their DL architecture has excellent performance (F1-score $>80 \%$ ) in detecting NPA for scans of different disease severity and image quality as well as for ultra-widefield OCTA. This result highlighted the potential clinical applications of a DL configuration for the early detection and progression assessment of DR [90]. Furthermore, to address the problem of signal reduction artefacts in the detection and quantification of NPA in OCTA, Guo et al. successfully updated their DL architecture to distinguish NPA from signal reduction artefacts. The network achieved both strong specificity and sensitivity for NPA detection across a wide range of DR severity and scan quality (dice coefficient $>0.87$ ) [91]. In recent years, other DL models have been developed for OCTA image quality assessment [92], object segmentation [93], and quantification [94]. Notably, through the extreme learning machine, one experiment based on tetragonal local OCTA pattern features has provided the precise identification of different severity of the DR in an efficient manner [95]. Considering the prevalence of DM and the heavy burden caused by DR, the implementation of DL will play a more critical role in the OCTA image analysis. More solid related DL-OCTA research could be awaiting.

\section{Conclusions}

The future in application of OCTA technology for assessing DR is promising. OCTA may potentially serve as an integral tool in DR management in tertiary care. The qualitative assessment of vascular changes in varying stages of DR has improved our understanding of the pathophysiology of DR. Quantitative OCTA measurements can objectively assess retinal microvascular changes, highlighting its potential to be used as a standardized and automated tool for detection and monitoring of DR. Furthermore, the capability of OCTA to detect clinical onset of DR and prediction for its progression may become useful toward individualised management of diabetic eye disease.

\section{Compliance with ethical standards}

Financial support CUHK Direct Grant (Reference No. 2015.1.046 \& 2017.054).

Conflict of interest The authors declare that they have no conflict of interest.

Publisher's note Springer Nature remains neutral with regard to jurisdictional claims in published maps and institutional affiliations.

\section{References}

1. Ogurtsova K, da Rocha Fernandes J, Huang Y, Linnenkamp U, Guariguata L, Cho NH, et al. IDF diabetes atlas: global estimates for the prevalence of diabetes for 2015 and 2040. Diabetes Res Clin Pract. 2017;128:40-50.

2. Wong TY, Cheung CM, Larsen M, Sharma S, Simo R. Diabetic retinopathy. Nat Rev Dis Prim. 2016;2:16012.

3. Jampol LM, Glassman AR, Sun J. Evaluation and care of patients with diabetic retinopathy. N Engl J Med. 2020;382:1629-37.

4. Spaide RF. Optical coherence tomography angiography signs of vascular abnormalization with antiangiogenic therapy for choroidal neovascularization. Am J Ophthalmol. 2015;160:6-16.

5. Spaide RF, Fujimoto JG, Waheed NK, Sadda SR, Staurenghi G. Optical coherence tomography angiography. Prog Retin Eye Res. 2018;64:1-55.

6. Novotny HR, Alvis DL. A method of photographing fluorescence in circulating blood in the human retina. Circulation 1961;24:82-6.

7. Spaide RF, Klancnik JM, Cooney MJ. Retinal vascular layers imaged by fluorescein angiography and optical coherence tomography angiography. JAMA Ophthalmol. 2015;133:45-50.

8. Weinhaus RS, Burke JM, Delori FC, Snodderly DM. Comparison of fluorescein angiography with microvascular anatomy of macaque retinas. Exp eye Res. 1995;61:1-16.

9. Mendis KR, Balaratnasingam C, Yu P, Barry CJ, McAllister IL, Cringle SJ, et al. Correlation of histologic and clinical images to determine the diagnostic value of fluorescein angiography for studying retinal capillary detail. Investig Ophthalmol Vis Sci. 2010;51:5864-9.

10. Johnson RN, Fu AD, McDonald HR, Jumper JM, Ai E, Cunningham ET, et al. Fluorescein angiography: basic principles and interpretation. InRetina Fifth Edition. Elsevier Inc. 2012.

11. Klein R, Meuer SM, Moss SE, Klein BE. Retinal microaneurysm counts and 10-year progression of diabetic retinopathy. Arch Ophthalmol. 1995;113:1386-91.

12. Klein R, Meuer SM, Moss SE, Klein BE. The relationship of retinal microaneurysm counts to the 4-year progression of diabetic retinopathy. Arch Ophthalmol. 1989;107:1780-5.

13. Ribeiro ML, Nunes SG, Cunha-Vaz JG. Microaneurysm turnover at the macula predicts risk of development of clinically significant macular edema in persons with mild nonproliferative diabetic retinopathy. Diabetes Care. 2013;36:1254-9.

14. Thompson IA, Durrani AK, Patel S. Optical coherence tomography angiography characteristics in diabetic patients without clinical diabetic retinopathy. Eye. 2019;33:648-52. 
15. Ishibazawa A, Nagaoka T, Takahashi A, Omae T, Tani T, Sogawa $\mathrm{K}$, et al. Optical coherence tomography angiography in diabetic retinopathy: a prospective pilot study. Am J Ophthalmol. 2015;160:35-44. e1.

16. Schwartz DM, Fingler J, Kim DY, Zawadzki RJ, Morse LS, Park SS, et al. Phase-variance optical coherence tomography: a technique for noninvasive angiography. Ophthalmology. 2014;121:180-7.

17. Miwa Y, Murakami T, Suzuma K, Uji A, Yoshitake S, Fujimoto $\mathrm{M}$, et al. Relationship between functional and structural changes in diabetic vessels in optical coherence tomography angiography. Sci Rep. 2016;6:29064.

18. Yu S, Lu J, Cao D, Liu R, Liu B, Li T, et al. The role of optical coherence tomography angiography in fundus vascular abnormalities. BMC Ophthalmol. 2016;16:107.

19. Hamada M, Ohkoshi K, Inagaki K, Ebihara N, Murakami A. Visualization of microaneurysms using optical coherence tomography angiography: comparison of OCTA en face, OCT B-scan, OCT en face, FA, and IA images. Jpn J Ophthalmol. 2018;62:168-75.

20. Couturier A, Mané V, Bonnin S, Erginay A, Massin P, Gaudric A, et al. Capillary plexus anomalies in diabetic retinopathy on optical coherence tomography angiography. Retina. 2015;35:2384-91.

21. Hwang TS, Jia Y, Gao SS, Bailey ST, Lauer AK, Flaxel CJ, et al. Optical coherence tomography angiography features of diabetic retinopathy. Retin. 2015;35:2371.

22. Stitt A, Gardiner T, Archer D. Histological and ultrastructural investigation of retinal microaneurysm development in diabetic patients. Br J Ophthalmol. 1995;79:362-7.

23. Parravano M, De Geronimo D, Scarinci F, Querques L, Virgili G, Simonett JM, et al. Diabetic microaneurysms internal reflectivity on spectral-domain optical coherence tomography and optical coherence tomography angiography detection. Am J Ophthalmol. 2017:179:90-6.

24. Gangnon RE, Davis MD, Hubbard LD, Aiello LM, Chew EY, Ferris FL, et al. A severity scale for diabetic macular edema developed from ETDRS data. Investig Ophthalmol Vis Sci. 2008;49:5041-7.

25. Pan J, Chen D, Yang X, Zou R, Zhao K, Cheng D, et al. Characteristics of neovascularization in early stages of proliferative diabetic retinopathy by optical coherence tomography angiography. Am J Ophthalmol. 2018;192:146-56.

26. Schaal KB, Munk MR, Wyssmueller I, Berger LE, Zinkernagel MS, Wolf S. Vascular abnormalities in diabetic retinopathy assessed with swept-source optical coherence tomography angiography widefield imaging. Retina. 2019;39:79-87.

27. Shimouchi A, Ishibazawa A, Ishiko $S$, Omae $T$, Ro-Mase $T$, Yanagi $\mathrm{Y}$, et al. A proposed classification of intraretinal microvascular abnormalities in diabetic retinopathy following panretinal photocoagulation. Investig Ophthalmol Vis Sci. 2020;61:34.

28. de Carlo TE, Bonini Filho MA, Baumal CR, Reichel E, Rogers A, Witkin AJ, et al. Evaluation of preretinal neovascularization in proliferative diabetic retinopathy using optical coherence tomography angiography. Ophthalmic Surg, lasers imaging Retin. 2016;47:115-9.

29. Bandello F, Corbelli E, Carnevali A, Pierro L, Querques G Optical coherence tomography angiography of diabetic retinopathy. OCT angiography in retinal and macular diseases. 56: Karger Publishers; 2016. p. 107-12.

30. Group ETDRSR. Fundus photographic risk factors for progression of diabetic retinopathy: ETDRS report number 12. Ophthalmology 1991;98:823-33.

31. Khalid H, Schwartz R, Nicholson L, Huemer J, El-Bradey MH, Sim DA, et al. Widefield optical coherence tomography angiography for early detection and objective evaluation of proliferative diabetic retinopathy. Br J Ophthalmol. 2020.
32. Jia Y, Bailey ST, Hwang TS, McClintic SM, Gao SS, Pennesi $\mathrm{ME}$, et al. Quantitative optical coherence tomography angiography of vascular abnormalities in the living human eye. Proc Natl Acad Sci. 2015;112:E2395-E402.

33. Tang FY, Ng DS, Lam A, Luk F, Wong R, Chan C, et al. Determinants of quantitative optical coherence tomography angiography metrics in patients with diabetes. Sci Rep. 2017;7:1-10

34. Chui TY, Zhong Z, Song H, Burns SA. Foveal avascular zone and its relationship to foveal pit shape. Optom Vis Sci. 2012;89:602.

35. Bresnick GH, Condit R, Syrjala S, Palta M, Groo A, Korth K. Abnormalities of the foveal avascular zone in diabetic retinopathy. Arch Ophthalmol. 1984;102:1286-93.

36. Shiihara H, Terasaki H, Sonoda S, Kakiuchi N, Shinohara Y, Tomita M, et al. Objective evaluation of size and shape of superficial foveal avascular zone in normal subjects by optical coherence tomography angiography. Sci Rep. 2018;8:1-9.

37. Samara WA, Say EA, Khoo CT, Higgins TP, Magrath G, Ferenczy $\mathrm{S}$, et al. Correlation of foveal avascular zone size with foveal morphology in normal eyes using optical coherence tomography angiography. Retina. 2015;35:2188-95.

38. You QS, Freeman WR, Weinreb RN, Zangwill L, Manalastas PIC, Saunders LJ, et al. Reproducibility of vessel density measurement with optical coherence tomography angiography in eyes with and without retinopathy. Retin. 2017;37:1475.

39. Durbin MK, An L, Shemonski ND, Soares M, Santos T, Lopes M, et al. Quantification of retinal microvascular density in optical coherence tomographic angiography images in diabetic retinopathy. JAMA Ophthalmol. 2017;135:370-6.

40. Dimitrova G, Chihara E, Takahashi H, Amano H, Okazaki K. Quantitative retinal optical coherence tomography angiography in patients with diabetes without diabetic retinopathy. Investig Ophthalmol Vis Sci. 2017;58:190-6.

41. Lim HB, Kim YW, Kim JM, Jo YJ, Kim JY. The importance of signal strength in quantitative assessment of retinal vessel density using optical coherence tomography angiography. Sci Rep. 2018;8:1-8.

42. Chu Z, Lin J, Gao C, Xin C, Zhang Q, Chen C-L, et al. Quantitative assessment of the retinal microvasculature using optical coherence tomography angiography. J Biomed Opt. 2016;21:066008.

43. Hirano T, Kitahara J, Toriyama Y, Kasamatsu H, Murata T, Sadda S. Quantifying vascular density and morphology using different swept-source optical coherence tomography angiographic scan patterns in diabetic retinopathy. $\mathrm{Br} \mathrm{J}$ Ophthalmol. 2019;103:216-21.

44. Uji A, Balasubramanian S, Lei J, Baghdasaryan E, Al-Sheikh M, Sadda SR. Impact of multiple en face image averaging on quantitative assessment from optical coherence tomography angiography images. Ophthalmology. 2017;124:944-52.

45. Cheung CY-, Zheng Y, Hsu W, Lee ML, Lau QP, Mitchell P, et al. Retinal vascular tortuosity, blood pressure, and cardiovascular risk factors. Ophthalmology. 2011;118:812-8.

46. MacGillivray TJ, Cameron JR, Zhang Q, El-Medany A, Mulholland C, Sheng Z, et al. Suitability of UK Biobank retinal images for automatic analysis of morphometric properties of the vasculature. PLoS ONE. 2015;10:e0127914.

47. Sasongko MB, Wong TY, Nguyen TT, Cheung CY, Shaw JE, Wang JJ. Retinal vascular tortuosity in persons with diabetes and diabetic retinopathy. Diabetologia. 2011;54:2409-16.

48. Cao D, Yang D, Huang Z, Zeng Y, Wang J, Hu Y, et al. Optical coherence tomography angiography discerns preclinical diabetic retinopathy in eyes of patients with type 2 diabetes without clinical diabetic retinopathy. Acta Diabetologica. 2018;55:469-77.

49. Alibhai AY, Moult EM, Shahzad R, Rebhun CB, Moreira-Neto C, McGowan M, et al. Quantifying microvascular changes using 
OCT angiography in diabetic eyes without clinical evidence of retinopathy. Ophthalmology. Retina. 2018;2:418-27.

50. Talisa E, Chin AT, Bonini Filho MA, Adhi M, Branchini L, Salz DA, et al. Detection of microvascular changes in eyes of patients with diabetes but not clinical diabetic retinopathy using optical coherence tomography angiography. Retina. 2015;35:2364-70.

51. Choi W, Waheed NK, Moult EM, Adhi M, Lee B, De TC, et al. Ultrahigh speed swept source optical coherence tomography angiography of retinal and choriocapillaris alterations in diabetic patients with and without retinopathy. Retina. 2017;37:11-21.

52. Takase N, Nozaki M, Kato A, Ozeki H, Yoshida M, Ogura Y. Enlargement of foveal avascular zone in diabetic eyes evaluated by en face optical coherence tomography angiography. Retina. 2015;35:2377-83.

53. Sun Z, Tang F, Wong R, Lok J, Szeto SK, Chan JC, et al. OCT angiography metrics predict progression of diabetic retinopathy and development of diabetic macular edema: a prospective study. Ophthalmology. 2019;126:1675-84.

54. Kim AY, Chu Z, Shahidzadeh A, Wang RK, Puliafito CA, Kashani AH. Quantifying microvascular density and morphology in diabetic retinopathy using spectral-domain optical coherence tomography angiography. Investig Ophthalmol Vis Sci. 2016;57: OCT362-OCT70.

55. Nesper PL, Roberts PK, Onishi AC, Chai H, Liu L, Jampol LM, et al. Quantifying microvascular abnormalities with increasing severity of diabetic retinopathy using optical coherence tomography angiography. Investig Ophthalmol Vis Sci. 2017;58: BIO307-BIO15.

56. Onishi AC, Nesper PL, Roberts PK, Moharram GA, Chai H, Liu $\mathrm{L}$, et al. Importance of considering the middle capillary plexus on OCT angiography in diabetic retinopathy. Investig Ophthalmol Vis Sci. 2018;59:2167-76.

57. Tang FY, Chan EO, Sun Z, Wong R, Lok J, Szeto S, et al. Clinically relevant factors associated with quantitative optical coherence tomography angiography metrics in deep capillary plexus in patients with diabetes. Eye Vis. 2020;7:7.

58. Samara WA, Shahlaee A, Adam MK, Khan MA, Chiang A, Maguire JI, et al. Quantification of diabetic macular ischemia using optical coherence tomography angiography and its relationship with visual acuity. Ophthalmology 2017;124:235-44.

59. Lei J, Yi E, Suo Y, Chen C, Xu X, Ding W, et al. Distinctive analysis of macular superficial capillaries and large vessels using optical coherence tomographic angiography in healthy and diabetic eyes. Investig Ophthalmol Vis Sci. 2018;59:1937-43.

60. Ting DSW, Tan GSW, Agrawal R, Yanagi Y, Sie NM, Wong $\mathrm{CW}$, et al. Optical coherence tomographic angiography in type 2 diabetes and diabetic retinopathy. JAMA Ophthalmol. 2017;135:306-12.

61. Lee H, Lee M, Chung H, Kim HC. Quantification of retinal vessel tortuosity in diabetic retinopathy using optical coherence tomography angiography. Retina 2018;38:976-85.

62. Johannesen SK, Viken JN, Vergmann AS, Grauslund J. Optical coherence tomography angiography and microvascular changes in diabetic retinopathy: a systematic review. Acta Ophthalmologica. 2019;97:7-14.

63. PhD DYYM, PhD SJC, PhD ENSM, PhD PKY, Fracp GJ, Fracp MEC. Pathogenesis and intervention strategies in diabetic retinopathy. Clin Exp Ophthalmol. 2001;29:164-6.

64. Bek T. Transretinal histopathological changes in capillary-free areas of diabetic retinopathy. Acta ophthalmologica. 1994;72:409-15.

65. Lee J, Moon BG, Cho AR, Yoon YH. Optical coherence tomography angiography of DME and its association with anti-VEGF treatment response. Ophthalmology. 2016;123:2368-75.

66. Toto L, D'Aloisio R, Di Nicola M, Di Martino G, Di Staso S, Ciancaglini $\mathrm{M}$, et al. Qualitative and quantitative assessment of vascular changes in diabetic macular edema after dexamethasone implant using optical coherence tomography angiography. Int $\mathrm{J}$ Mol Sci. 2017;18:1181.

67. de Carlo TE, Chin AT, Joseph T, Baumal CR, Witkin AJ, Duker JS, et al. Distinguishing diabetic macular edema from capillary nonperfusion using optical coherence tomography angiography. Ophthalmic Surgery, Lasers and Imaging. Retina 2016;47:108-14.

68. Iwase T, Yamamoto K, Kobayashi M, Ra E, Murotani K, Terasaki $\mathrm{H}$. What ocular and systemic variables affect choroidal circulation in healthy eyes. Medicine. 2016;95.

69. Conti FF, Qin VL, Rodrigues EB, Sharma S, Rachitskaya AV, Ehlers JP, et al. Choriocapillaris and retinal vascular plexus density of diabetic eyes using split-spectrum amplitude decorrelation spectral-domain optical coherence tomography angiography. Br J Ophthalmol. 2019;103:452-6.

70. Olver J. Functional anatomy of the choroidal circulation: methyl methacrylate casting of human choroid. Eye 1990;4:262-72.

71. Zheng F, Zhang Q, Shi Y, Russell JF, Motulsky EH, Banta JT, et al. Age-dependent changes in the macular choriocapillaris of normal eyes imaged with swept-source optical coherence tomography angiography. Am J Ophthalmol. 2019;200:110-22.

72. Nassisi M, Baghdasaryan E, Tepelus T, Asanad S, Borrelli E, Sadda SR. Topographic distribution of choriocapillaris flow deficits in healthy eyes. PLoS ONE. 2018;13:e0207638.

73. Dai Y, Zhou H, Chu Z, Zhang Q, Chao JR, Rezaei KA, et al. Microvascular changes in the choriocapillaris of diabetic patients without retinopathy investigated by swept-source OCT angiography. Investig Ophthalmol Vis Sci. 2020;61:50.

74. McLeod DS, Lutty GA. High-resolution histologic analysis of the human choroidal vasculature. Investig Ophthalmol Vis Sci. 1994;35:3799-811.

75. Alnawaiseh M, Rosentreter A, Hillmann A, Alex AF, Niekämper $\mathrm{D}$, Heiduschka $\mathrm{P}$, et al. OCT angiography in the mouse: a novel evaluation method for vascular pathologies of the mouse retina. Exp Eye Res. 2016;145:417-23.

76. Uehara H, Lesuma T, Stocking P, Jensen N, Kumar SR, Zhang MA, et al. Detection of microvascular retinal changes in type I diabetic mice with optical coherence tomography angiography. Exp Eye Res. 2019;178:91-8.

77. Giannakaki-Zimmermann H, Kokona D, Wolf S, Ebneter A, Zinkernagel MS. Optical coherence tomography angiography in mice: comparison with confocal scanning laser microscopy and fluorescein angiography. Transl Vis Sci Technol. 2016;5:11.

78. Holmen IC, Konda SM, Pak JW, McDaniel KW, Blodi B, Stepien KE, et al. Prevalence and severity of artifacts in optical coherence tomographic angiograms. JAMA Ophthalmol. 2020; 138:119-26.

79. Kashani AH, Chen C-L, Gahm JK, Zheng F, Richter GM, Rosenfeld PJ, et al. Optical coherence tomography angiography: A comprehensive review of current methods and clinical applications. Prog Retinal Eye Res. 2017;60:66-100.

80. Hormel TT, Jia Y, Jian Y, Hwang TS, Bailey ST, Pennesi ME, et al. Plexus-specific retinal vascular anatomy and pathologies as seen by projection-resolved optical coherence tomographic angiography. Progress in Retinal and Eye Research. 2020:100878.

81. Ting DSW, Pasquale LR, Peng L, Campbell JP, Lee AY, Raman $\mathrm{R}$, et al. Artificial intelligence and deep learning in ophthalmology. Br J Ophthalmol. 2019;103:167-75.

82. LeCun Y, Bengio Y, Hinton G. Deep learning. Nature. 2015;521:436-44.

83. Sheikh HR, Sabir MF, Bovik AC. A statistical evaluation of recent full reference image quality assessment algorithms. IEEE Trans Image Process. 2006;15:3440-51.

84. Lakhani P, Sundaram B. Deep learning at chest radiography: automated classification of pulmonary tuberculosis by using convolutional neural networks. Radiology 2017;284:574-82. 
85. Balyen L, Peto T. Promising artificial intelligence-machine learning-deep learning algorithms in ophthalmology. Asia Pac J Ophthalmol. 2019;8:264-72.

86. Ran AR, Cheung CY, Wang X, Chen H, Luo L-y, Chan PP, et al. Detection of glaucomatous optic neuropathy with spectral-domain optical coherence tomography: a retrospective training and validation deep-learning analysis. Lancet Digital Health. 2019;1:e172-e82.

87. Ting DSW, Cheung CY-L, Lim G, Tan GSW, Quang ND, Gan A, et al. Development and validation of a deep learning system for diabetic retinopathy and related eye diseases using retinal images from multiethnic populations with diabetes. JAMA. 2017;318:2211-23.

88. Keenan TD, Dharssi S, Peng Y, Chen Q, Agrón E, Wong WT, et al. A deep learning approach for automated detection of geographic atrophy from color fundus photographs. Ophthalmology. 2019;126:1533-40.

89. Milea D, Najjar RP, Jiang Z, Ting D, Vasseneix C, Xu X, et al. Artificial intelligence to detect papilledema from ocular fundus photographs. N Engl J Med. 2020;382:1687-95.

90. Guo Y, Camino A, Wang J, Huang D, Hwang TS, Jia Y. MEDnet, a neural network for automated detection of avascular area in OCT angiography. Biomed Opt Express. 2018;9:5147-58.
91. Guo Y, Hormel TT, Xiong H, Wang B, Camino A, Wang J, et al. Development and validation of a deep learning algorithm for distinguishing the nonperfusion area from signal reduction artifacts on OCT angiography. Biomed Opt Express. 2019;10:3257-68.

92. Lauermann JL, Treder M, Alnawaiseh M, Clemens CR, Eter N, Alten F. Automated OCT angiography image quality assessment using a deep learning algorithm. Graefes Arch Clin Exp Ophthalmol. 2019;257:1641-8.

93. Prentasic P, Heisler M, Mammo Z, Lee S, Merkur A, Navajas E, et al. Segmentation of the foveal microvasculature using deep learning networks. J Biomed Opt. 2016;21:75008.

94. Guo M, Zhao M, Cheong AMY, Dai H, Lam AKC, Zhou Y. Automatic quantification of superficial foveal avascular zone in optical coherence tomography angiography implemented with deep learning. Vis Comput Ind Biomed Art. 2019;2:21.

95. Nazir T, Irtaza A, Shabbir Z, Javed A, Akram U, Mahmood MT. Diabetic retinopathy detection through novel tetragonal local octa patterns and extreme learning machines. Artif Intell Med. 2019;99:101695. 